\title{
Are Novel Oral Anticoagulants (Noacs) as Safe as They are Said to Be?
}

\section{Kamali F*}

Department of Human and Experimental Pharmacology, Institute of Cellular Medicine, The Medical School, Newcastle University, United Kingdom

Warfarin is the most commonly prescribed oral anticoagulant used for the treatment of thromboembolic disorders. Warfarin causes anticoagulation by inhibiting the recycling of the reduced form of vitamin $\mathrm{K}$ in the coagulation cascade; vitamin $\mathrm{K}$ is essential for the functionalization of the vitamin K-dependent clotting proteins, II, VII, IX and $\mathrm{X}$. It is notoriously difficult to predict clinical response to a fixed dose of warfarin. This is due to a number of reasons including the wide inter-individual variability in warfarin dose requirement, influenced by both genetic and environmental factors, the drug's narrow therapeutic index and its delayed effect [1]. As a result patients treated with warfarin require frequent laboratory monitoring of their International Normalised Ratio (INR) of the prothrombin time, and dose adjustment, in order to minimise risk of thrombosis (due to under-doing) or bleeding (due to over-dosing) [1].

Over the last decade the pharmaceutical industry has invested heavily in developing new agents that are safer than warfarin but as effective in treating thromboembolic disorders. Dabigatran (a direct thrombin inhibitor), rivaroxaban and apixaban (both direct factor Xa inhibitors) are part of the new generation of oral anticoagulants (NOACs) now recommended for stroke prevention in patients with non-valvular atrial fibrillation across Europe and the US.

The main advantages of NOACs over warfarin are their predictable pharmacokinetics and clinical response thus obviating the need for routine anticoagulation monitoring or monitoring of plasma drug levels, fewer drug interactions and the lack of interaction with diet. More crucially NOACS were developed and marketed to be used as fixed-dose regimens, which is considered to be a substantial advantage over warfarin. Prescription numbers for NOACs have risen sharply since their launch for both newly diagnosed patients requiring initiation of anticoagulant therapy and existing patients on warfarin with poor anticoagulation control being switched to a NOAC, with prescriptions in England for dabigatran in 2012 compared to 2011 increasing 1,600\%, from around 3,000 to around 48,000, and prescriptions for rivaroxaban in 2012 being around 16,000 [2].

Whilst non-inferiority of NOACs to warfarin in their clinical effectiveness for stroke prophylaxis in patients with atrial fibrillation in the RE-LY, ROCKET-AF, and ARISTOTLE studies has been established, the bleeding adverse reactions reported in those trials, may be contributed to by patient age, with the noted incidence of bleeding being greater in those aged 75 years and over [3-5]. It is of concern that in view of the use of NOACs in atrial fibrillation the more elderly were not well represented in the trials, and clinical experience is too short to provide models of use which optimise the benefits of therapy and ensure therapy can be safely prescribed. Although prescribing rates for NOACs have grown rapidly since their launch, concerns about fatal bleeds have emerged particularly in elderly people, who are at a greater risk. A later sub-analysis of the RE-LY trial data by the manufacturer of dabigatran, Boehringer Ingleheim, showed that there were 5-fold variations in plasma dabigatran concentration for each of the two doses $(110$ and $150 \mathrm{mg})$ that were tested [6]. Further analysis of the data showed that renal function is the most important determinant for plasma dabigatran concentration with age being the most important covariate. The analysis also showed that beyond a certain plasma concentration of the drug major bleeding events continued to increase as the plasma levels increased with little effect on rates of stroke and systemic embolism [6]. A most recent investigation by the BMJ revealed that the results of the sub-analysis were not disclosed by the company to the relevant authorities at the time of licence application [7]. The revelation regarding the nature of the undisclosed data raise further concerns about dabigatran safety and the claims that the drug shows little inter-individual variation in plasma levels, and that monitoring of plasma levels is not needed [7-9]. The RE-LY sub-group analysis results are based on data derived from a tightly controlled clinical trial where strict inclusion criteria were enforced. There could be an even greater variance in plasma drug concentration and subsequently a greater associated risk in uncontrolled older patients who are prescribed NOACs in the community at large. It is likely that the other NOACs (rivaroxaban and apixaban) also exhibit variability in blood concentrations and could therefore pose similar safety issues as those for dabigatran.

It is clear that NOACs have a role in the prophylaxis of stroke. However, in the light of the recent revelations about dabigatran it seems that more research will be needed to assess the safety of these drugs in atrial fibrillation patients particularly in the wider community.

\section{References}

1. Kamali F, Wynne H (2010) Pharmacogenetics of warfarin. Annu Rev Med 61:63-75.

2. Prescribing and Primary Care Services Health and Social Care Information Centre. Prescriptions Dispensed in the Community: England 2002-2012.

3. Connolly SJ, Ezekowitz MD, Yusuf S, Eikelboom J, Oldgren J, et al. (2009) Dabigatran versus warfarin in patients with atrial fibrillation. N Engl J Med 361: 1139-1151.

4. Granger CB, Alexander JH, McMurray JJ, Lopes RD, Hylek EM, et al. (2011) Apixaban versus warfarin in patients with atrial fibrillation. N Engl J Med. 365: 981-992.

5. Patel MR, Mahaffey KW, Garg J, Pan G, Singer DE, et al. (2011) Rivaroxaban versus warfarin in nonvalvular atrial fibrillation. $N$ Engl J Med 365: 883-891.

6. Reilly PA, Lehr T, Haertter S, Connolly SJ, Yusuf S, et al. (2014) The effect of dabigatran plasma concentrations and patient characteristics on the frequency of ischemic stroke and major bleeding in atrial fibrillation patients: the RE-LY Trial (Randomized Evaluation of Long-Term Anticoagulation Therapy). J Am Coll Cardiol 63: 321-328.

*Corresponding author: Kamali F, Human and Experimental Pharmacology Institute of Cellular Medicine, The Medical School, Newcastle University, United Kingdom, E-mail: farhad.kamali@ncl.ac.uk

Received October 14, 2014; Accepted October 15, 2014; Published October 17 2014

Citation: Kamali F (2014) Are Novel Oral Anticoagulants (Noacs) as Safe as They are Said to Be? Adv Pharmacoepidemiol Drug Saf 3: e126. doi:10.4172/21671052.1000e126

Copyright: () 2014 Kamali F. This is an open-access article distributed under the terms of the Creative Commons Attribution License, which permits unrestricted use, distribution, and reproduction in any medium, provided the original author and source are credited. 
Citation: Kamali F (2014) Are Novel Oral Anticoagulants (Noacs) as Safe as They are Said to Be? Adv Pharmacoepidemiol Drug Saf 3: e126. doi:10.4172/2167-1052.1000e126

Page 2 of 2

7. Cohen D (2014) Dabigatran: how the drug company withheld important analyses. BMJ 349: g4670.

8. Charlton B and Redberg R (2014) The trouble with dabigatran. BMJ 349: g4681.
9. Moore TJ, Cohen MR, Mattison DR (2014) Dabigatran, bleeding, and the regulators. BMJ 349: g4517. 